Mitteilungen der Österreichischen Geographischen Gesellschaft,

156. Jg. (Jahresband), Wien 2014, S. 273-292

\title{
Kulturelle Stadtentwicklung an der Wiener Peripherie
}

\author{
Walter RoHN, Wien*
}

mit 2 Abb. und 1 Tab. im Text

\section{INHALT}

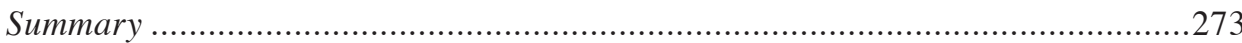

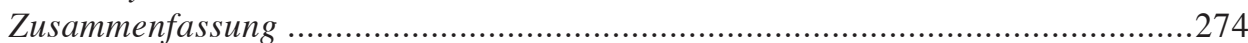

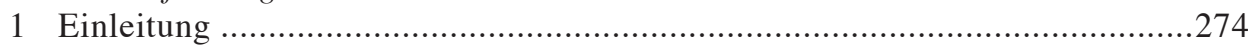

2 Theoretische und methodische Ansätze ..................................................275

3 Neue Kulturinitiativen in den Wiener Randbezirken ................................277

4 Übergeordnete Fragen zur Kultur am Wiener Stadtrand ..............................281

5 Kultur im 20. Pariser Arrondissement ...........................................................283

6 Wiener und Pariser Randbezirke im Vergleich ...........................................285

7 Effekte von Kulturprojekten auf die Entwicklung peripherer Stadtteile ..........286

8 Kulturpolitik für städtische Randzonen ..................................................28

9 Förderung dezentraler Kulturinitiativen .................................................289

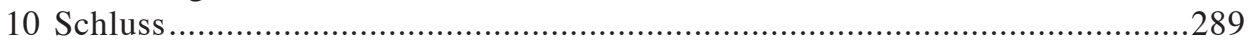

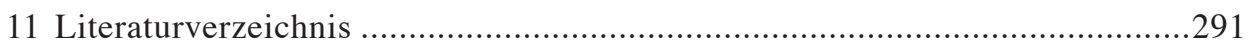

\section{Summary}

\section{Cultural urbanism on the outskirts of Vienna}

Cultural facilities, such as theatres, cinemas, galleries, music venues etc., are in a valuable position to stimulate the development of urban fringe areas. The first part of this paper presents an overview of new cultural facilities on the outskirts of Vienna [Wien] and summarises recent developments in this field. This is followed by an overview of cultural facilities in a fringe district of Paris and a brief comparison between the studied districts on the outskirts of Vienna and Paris. The second section focuses on the results of interviews with experts in the fields of culture, urban development and city administration. The experts are united in their belief that cultural facilities can foster the cultural, urbanistic, economic and social development of urban fringe areas. Based on this, an outline for a new cultural policy in Vienna's peripheral dis-

* Dr. Walter Rohn, Institut für Stadt- und Regionalforschung, Österreichische Akademie der Wissenschaften, Postgasse 7/4/2, A-1010 Wien; E-Mail: walter.rohn@oeaw.ac.at, http://www.oeaw.ac.at/isr 
tricts is drawn and practical measures in support of these areas are discussed. The paper concludes with some concrete recommendations for Vienna.

\section{Zusammenfassung}

Kulturinitiativen wie Theater, Kinos, Galerien, Musikclubs usw. können wertvolle Impulse für die Entwicklung städtischer Randgebiete setzen. Der erste Teil dieses Beitrags gibt einen Überblick über neue Kultureinrichtungen in den Wiener Außenbezirken und fasst die rezenten Entwicklungen in diesem Bereich zusammen. In der Folge wird ein Blick auf die Kulturszene eines Pariser Randbezirks geworfen und ein kurzer Vergleich zwischen den in Wien und Paris untersuchten Peripheriebezirken angestellt. Im zweiten Teil werden die Ergebnisse einer Befragung von Experten in den Bereichen Kultur, Stadtentwicklung und Verwaltung zusammengefasst. Die Experten stimmen darin überein, dass Kulturinitiativen die kulturelle, städtebauliche, wirtschaftliche und soziale Entwicklung städtischer Randgebiete stimulieren können. Anschließend werden die Konturen einer neuen Kulturpolitik für die Wiener Außenbezirke gezeichnet und praktische Maßnahmen zur Förderung dieser Gebiete diskutiert. Der Beitrag schließt mit einigen Empfehlungen für Wien.

\section{Einleitung}

Kulturprojekte sind in der Lage, effiziente Beiträge zur Stadtentwicklung zu leisten. Auf gesamtstädtischer Ebene dienen dazu große Kulturbauten wie Opernhäuser, Konzerthäuser, Museen und Bibliotheken. Role Model der kulturellen Stadtentwicklung ist die an der Ostküste der USA gelegene Stadt Baltimore, die bereits in den späten 1970er Jahren derartige Initiativen setzte. Prominente europäische Beispiele sind die in den 1980er Jahren in Paris errichteten Grands Travaux (Cité des Sciences et de 1'Industrie, Musée d'Orsay, Institut du Monde Arabe etc.), Frank Gherys 1997 in Bilbao eröffnetes Guggenheim-Museum und die 2000 in London inaugurierte Tate Modern (Roнn 2013, S. 17f.). Kulturprojekte können aber auch auf kleinräumiger Ebene, d.h. in einzelnen Stadtvierteln, wertvolle Impulse für die Stadtentwicklung setzen. Dieser Aspekt steht im Mittelpunkt der folgenden Ausführungen.

Der Beitrag basiert auf den Ergebnissen des mehrjährigen Forschungsprojekts „Die neue Kultur am Rand der Städte“, das sich mit rezenten Kulturprojekten in den Randzonen europäischer Metropolen und den diesbezüglichen kulturpolitischen Ansätzen der jeweiligen Stadtverwaltungen befasste. Im Sinne einer Schwerpunktsetzung werden im Folgenden die Forschungsergebnisse zu Wien präsentiert, und es wird ein vergleichender Blick auf die Entwicklungen in einem Pariser Randbezirk geworfen. Das Forschungsprojekt wurde am Institut für Stadt- und Regionalforschung der Österreichischen Akademie der Wissenschaften durchgeführt und dankenswerterweise vom Kulturamt der Stadt Wien (MA7) gefördert. 
Im Fokus des Projekts standen folgende Forschungsfragen:

- Durch welche grundlegenden Merkmale sind Kulturinitiativen in städtischen Randzonen charakterisiert?

- Welche Beiträge können Kulturprojekte zur positiven Entwicklung urbaner Randgebiete leisten?

- In welcher Form kann städtische Kulturpolitik zur Förderung dezentraler Kulturinitiativen entworfen werden?

Zunächst sei ein Überblick über den Inhalt dieses Beitrags gegeben. Im anschlieBenden zweiten Kapitel werden die theoretischen und methodischen Grundlagen der Untersuchung zusammengefasst. Im Zentrum des dritten Abschnitts stehen die in jüngerer Vergangenheit in den Wiener Außenbezirken gegründeten Kultureinrichtungen. Übergeordnete Fragen zur Kultur am Wiener Stadtrand werden im vierten Kapitel behandelt. Der fünfte Abschnitt widmet sich der Kulturszene des 20. Pariser Arrondissements und im sechsten Kapitel werden die kulturellen Infrastrukturen von Wiener und Pariser Randbezirken verglichen. Die möglichen positiven Auswirkungen von Kulturprojekten auf die städtische Peripherie werden im siebenten Abschnitt zusammengefasst. Die Kapitel acht und neun behandeln die Umrisse einer möglichen neuen Kulturpolitik für die Wiener Außenbezirke und konkrete Fördermaßnahmen für die Gebiete am Stadtrand. Im abschließenden zehnten Kapitel werden einige Empfehlungen ausgesprochen.

\section{Theoretische und methodische Ansätze}

Grundlage der weiteren Ausführungen bildet ein breiter, anthropologischer Kulturbegriff wie ihn die Organisation der Vereinten Nationen für Erziehung, Wissenschaft und Kultur [United Nations Educational, Scientific and Cultural Organisation, UNESCO] verwendet. Sie definiert Kultur als die eine Gesellschaft oder soziale Gruppe kennzeichnende Gesamtheit von geistigen, materiellen, intellektuellen und emotionalen Merkmalen. Diese umfasst nicht nur die Kunst, sondern auch Lebensformen, grundlegende Menschenrechte, Wertesysteme, Traditionen und religiöse Überzeugungen (Municipial Cultural Planning Project 2003).

$\mathrm{Zu}$ definieren sind an dieser Stelle auch die im Mittelpunkt des Aufsatzes stehenden Kulturinitiativen. Gemäß der in der Fachliteratur verwendeten Diktion (KonRAD 2011, S. 148ff.) werden darunter Veranstaltungsorte und Festivals für Musik, Theater, Kabarett, Literatur, Bildende Kunst, Film und mehrere Kunstsparten umfassende Programme verstanden.

Als theoretischer Ansatz zur kulturellen Stadtentwicklung wird die Konzeption der kulturellen Planung (Cultural Planning) von Franco Bianchini und Lia GHILARDi (2004) gewählt. Die Strategie des Cultural Planning bietet eine ganzheitliche und räumliche Definition der kulturellen Ressourcen eines Ortes (Stadtteil, Stadt usw.). Zu diesen zählen unter anderem die Kunst, die Medien und das kulturelle Erbe, die Kulturen von 
Jugendlichen und ethnischen Minderheiten, lokale Traditionen, die Wahrnehmungen eines Ortes, die Topographie und die Qualitäten der natürlichen und gebauten Umwelt, die Vielfalt von Freizeit- und Unterhaltungseinrichtungen sowie das Ensemble von lokalen Produkten und Fertigkeiten. Durch die umfassende Inwertsetzung dieses im öffentlichen und privaten Sektor bestehenden kulturellen Reichtums soll die integrierte Entwicklung eines Territoriums ermöglicht werden.

Gemäß der Konzeption von Bianchini \& Ghilardi (2004) werden die kulturellen Ressourcen ins Zentrum gerückt und Zweiweg-Beziehungen zwischen diesen und anderen Bereichen öffentlicher Politik wie beispielsweise Architektur, Stadtentwicklung, Wohnungswesen, Bildung und Sozialwesen hergestellt. Die kulturellen Ressourcen dienen dabei als Katalysator für Entwicklungen in den angeführten Bereichen. Wesentlich ist das Verständnis der beschriebenen Strategie als kultureller Zugang zu Stadtentwicklung und kommunaler Politik.

Den spezifischen Ansatz für private und institutionelle Akteure repräsentiert die Akteur-Netzwerk-Theorie des französischen Soziologen Bruno Latour. In seinem Werk „Eine neue Soziologie für eine neue Gesellschaft“ geht Latour (2007) davon aus, dass die Existenzformen (in etwa: Rahmenbedingungen) das Handeln tragen. Das von anderen Handlungsträgern mitgeprägte Handeln stuft LATOUR als einen „Knoten, eine Schlinge, ein Konglomerat aus vielen überraschenden Handlungsquellen“ ein (LAtour 2007, S. 77). Die Handlungsträger werden als Akteure bezeichnet. Eine Besonderheit des Ansatzes von LATOuR besteht darin, dass er Menschen und Objekte, die eine Figuration aufweisen, als Akteure definiert. Als Figuration bezeichnet der französische Soziologe den Vorgang, einem Akteur eine Konsistenz, Gestalt oder Form zu verleihen. LATOUR vergleicht die Akteure mit Schauspielern auf der Bühne und führt als wesentliche Bestimmungsmerkmale eines Akteurs an, dass dieser einen Unterschied im Handeln anderer Akteure bewirkt, eine Veränderung hervorruft und Spuren hinterlässt.

Kevin Mulcahy (2006, S. 2) definiert Kulturpolitik als die Summe der Aktivitäten einer Regierung, Stadtverwaltung usw. in Bezug auf die Künste, die Kulturwirtschaft, den geisteswissenschaftlichen Bereich und das kulturelle Erbe. Dorte Sкот-Hansen (2005, S. 36) steckt den Rahmen einer kommunalen Kulturpolitik ab, indem sie deren Aufgaben zu vier Handlungsfeldern zusammenfasst: Unter dem ersten Handlungsfeld Aufklärung subsumiert die Autorin Kunstproduktion, Bewahrung des kulturellen Erbes, Publikumsförderung und Kunsterziehung. Der zweite Bereich Wirtschaft umfasst Veranstaltungen, Festivals, Leitprojekte und Place Marketing. Unterhaltung bezieht sich drittens auf Themenparks und herkömmliche Parkanlagen, Spielflächen und Paraden. Community Arts, soziale und ethnische Kunst, Subkulturen usw. dienen viertens der Ermächtigung. In diesen vier Handlungsfeldern sind die Aufgaben städtischer Kulturpolitik treffend zusammengefasst.

Das methodische Konzept der Studie beruht auf zwei Säulen: erstens der ethnographischen Methode und zweitens dem Städtevergleich. Die wichtigsten im Rahmen der ethnographischen Methode eingesetzten Verfahren sind Feldforschung (Robert Ezra PARKs ,nosing around“), teilnehmende Beobachtung und qualitative Interviews 
(LinDNER 2007, S. 9; LÜDERs 2009, S. 39ff.). In Berlin, Paris, Wien und Zagreb wurden insgesamt 61 Tiefeninterviews geführt und ausgewertet. Mit dem Rüstzeug des Städtevergleichs bzw. der komparativen Stadtforschung (MIEGgelbrinK \& WIEST 2009; Löw 2008) werden einander Peripheriebezirke der vier genannten Metropolen gegenübergestellt und die jeweiligen kulturpolitischen Konzepte verglichen.

\section{Neue Kulturinitiativen in den Wiener Randbezirken}

Seit Ende der 1990er Jahre entstand in den Wiener Außenbezirken X bis XXIII ${ }^{1)}$ eine Reihe neuer Kultureinrichtungen. Diese „,neue Gründerzeit“ ging vom XVI. Bezirk aus und erfasste später in Form eines Spill-Over die weiteren Bezirke am Westgürtel, die heute den räumlichen Schwerpunkt neuer Kulturinitiativen in den Peripheriebezirken bilden. Darüber hinaus sind im Süden sowie im Nordosten und Osten Wiens neue Kulturprojekte entstanden. Abbildung 1 gibt einen Überblick über 40 neuere Kulturinitiativen in den Wiener Außenbezirken, die regelmäßig Veranstaltungen anbieten. Diese Einrichtungen widmen sich der Bildenden Kunst, dem Film, der Musik und dem Theater oder bieten mehrere Kunstsparten an. Die nicht zu den Außenbezirken zählenden Stadtteile sind in der Abbildung mit einem Grauton belegt.

Für die detaillierte Untersuchung rezenter Wiener Kulturinitiativen wurden nach dem Kriterium des kontrastierenden Vergleichs die drei Peripheriebezirke Ottakring (XVI.), Döbling (XIX.) und Floridsdorf (XXI.) ausgewählt. Zu den drei analysierten Bezirken ist Folgendes festzuhalten:

Der im Westen Wiens gelegene XVI. Gemeindebezirk Ottakring ist der kleinste der drei Untersuchungsbezirke und weist die zweithöchste Einwohnerzahl unter ihnen auf. Im sogenannten „Arbeiterbezirk“ Ottakring liegt der Anteil der Ausländer an der Wohnbevölkerung über und der Bildungsgrad der Bevölkerung unter dem Wiener Durchschnitt. Im zentrumsnahen Gebiet um den Brunnenmarkt und den Yppenplatz ist seit Ende der 1990er Jahre eine lebendige Künstlerszene entstanden, die als Keimzelle der Gründung neuer Kulturinitiativen in den Wiener Außenbezirken zu bezeichnen ist. Das kulturelle Highlight des Bezirks repräsentiert „Soho in Ottakring“, das ein biennales Kunstfestival und eine ganzjährige Veranstaltungsreihe vereint. Darüber hinaus fasst der Verein „Grundstein“ eine Vielzahl von Kulturprojekten zusammen. Im XVI. Bezirk haben die Kulturinitiativen jene kritische Masse erreicht, die erforderlich ist, um für die gesamte Entwicklung des Bezirks wirksam zu werden. Die Kultureinrichtungen des Bezirks bedienen einerseits das lokale Publikum und ziehen andererseits Besucher aus ganz Wien an. In den vergangenen Jahren konnte das Kulturbudget des Bezirks stark erhöht werden.

Der im Nordwesten Wiens situierte XIX. Bezirk Döbling ist durch die niedrigste Bevölkerungszahl und die zweitgrößte Fläche der Untersuchungseinheiten charakteri1) Unter Wiener Außenbezirken werden die Gemeindebezirke außerhalb des Gürtels und links der Donau
verstanden. 


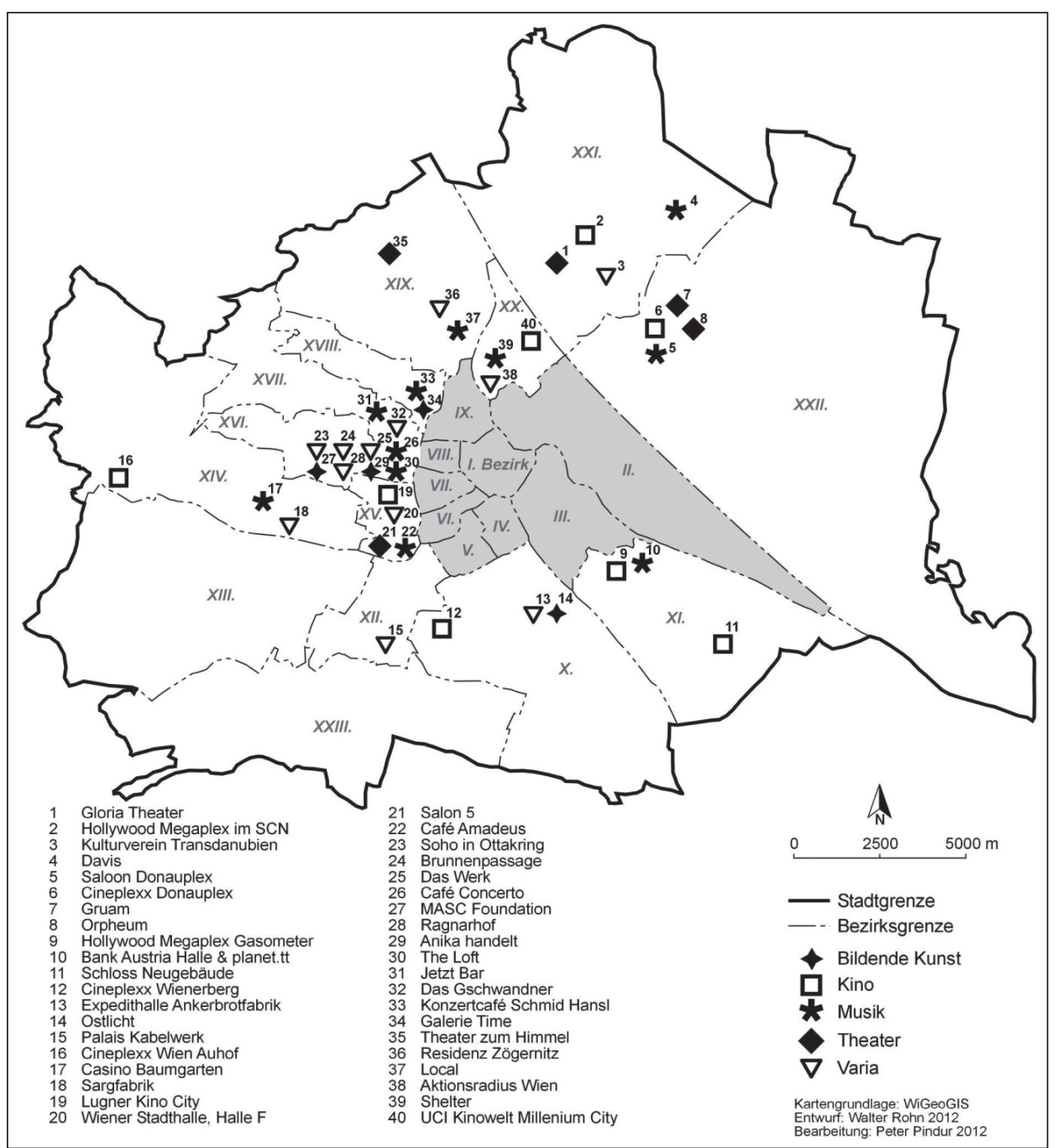

Quelle: Eigene Zusammenstellung auf Basis von Angaben in Printmedien und im Internet sowie eigenen Recherchen

Abb. 1: Rezente Kulturinitiativen in den Wiener Außenbezirken mit regelmäßigem Programm (2012)

siert. Dem Ausländeranteil nach ist der sogenannte „bürgerliche“ Bezirk Döbling unter und dem Bildungsgrad nach über dem Wiener Durchschnitt positioniert. Der Bezirk verfügt über ein engmaschiges Netz zumeist kleinerer Kulturinitiativen, das in erster Linie auf das lokale Publikum zugeschnitten ist. Mit dem „Theater zum Himmel“ und dem „Local“ besitzt Döbling nur zwei große neue Projekte. In jüngster Zeit konnte das Kulturbudget etwas erhöht werden. 
Im Norden Wiens gelegen, weist der sogenannte „Arbeiterbezirk“ Floridsdorf (XXI.) die größte Fläche und die größte Einwohnerzahl der drei Untersuchungseinheiten auf. Der Ausländeranteil des XXI. Bezirks und der Bildungsgrad seiner Bevölkerung liegen unter dem Durchschnitt der Bundeshauptstadt. Die vergleichsweise wenigen Kultureinrichtungen des Bezirks sind über eine große Fläche verteilt, was die Vernetzung erschwert. Das Angebot der Kulturinitiativen richtet sich vor allem an das lokale Publikum. Das „Gloria Theater“ und das „Hollywood Megaplex SCN“ repräsentieren die einzigen großen Kultureinrichtungen des Bezirks. Im Gegensatz zu den beiden anderen Untersuchungseinheiten blieb das Kulturbudget des XXI. Bezirks in den letzten Jahren unverändert.

Legt man die Aufwendungen der drei angeführten Bezirke für Kultur auf die jeweilige Einwohnerzahl um, so resultieren daraus folgende Maßzahlen: Pro Einwohner wendete der XVI. Bezirk im Jahr 2012 1,57 Euro an lokalen Kulturfördermitteln auf, der XIX. Bezirk 0,78 Euro und der XXI. Bezirk 0,39 Euro.

In den drei behandelten Bezirken wurden jeweils fünf Kulturprojekte analysiert. Zur besseren Übersicht erfolgte eine Klassifikation der 15 ausgewählten Kulturinitiativen nach verschiedenen Merkmalen (vgl. Tab. 1).

Mit der „Brunnenpassage“, dem „Werk“, dem „Ragnarhof“ und „Soho in Ottakring“ (alle XVI. Bezirk), dem „Local“ und dem „Theater zum Himmel“ (XIX.) sowie dem „Gloria Theater“ und dem „Hollywood Megaplex im SCN“ (XXI.) wurden acht Kulturinitiativen als groß eingestuft.

Sieben Einrichtungen sind auf jeweils eine Kunstsparte festgelegt: Das „Neue Theater in Döbling“, das „Theater zum Himmel“ (beide XIX.) und das „Gloria Theater“ (XXI.) widmen sich ausschließlich der Bühnenkunst. Das „Local“ (XIX.) und das „Davis“ (XXI.) sind auf Musik fokussiert; die „MASC Foundation“ (XVI.) forciert die Bildende Kunst und das „Hollywood Megaplex SCN“ (XXI.) präsentiert Kinofilme. Die weiteren acht Projekte befassen sich jeweils mit mehreren Kunstsparten. Eine breite Palette an Kunstformen bieten die „Brunnenpassage“, der „Ragnarhof“, „Soho in Ottakring“ und der „Kulturverein Transdanubien“ an. Somit werden von den Kulturprojekten alle gängigen Kunstsparten abgedeckt.

Die Projekte wurden mehrheitlich ab 1998 auf Initiative von Künstlern und Bezirksbewohnern gegründet. Institutionelle Akteure waren für die Gründung der „Brunnenpassage“, des „Forum 21“ und des „Hollywood Megaplex SCN“ verantwortlich. Die Mehrheit der Einrichtungen - auch größere Initiativen - wird nur in geringem Maß von der öffentlichen Hand unterstützt. Einer guten Förderung können sich lediglich die „Brunnenpassage“, „Soho in Ottakring“ und das „Gloria Theater“ erfreuen.

Den organisatorischen Rahmen für die künstlerischen Produktionen der meisten Kulturinitiativen bilden zumeist gemeinnützige Vereine. Bei einigen Projekten - zum Beispiel dem „Werk“ und dem „Davis“ - werden die gastronomischen Aktivitäten über privatwirtschaftliche Organisationsformen abgewickelt. Vollständig auf privatwirtschaftlicher Basis sind das „Local“ und das „Hollywood Megaplex SCN“ organisiert. Die 15 untersuchten Einrichtungen kooperieren mit anderen Initiativen im jeweiligen 


\begin{tabular}{|c|c|c|c|c|c|}
\hline $\begin{array}{l}\text { Untersuchte Kultur- } \\
\text { initiativen nach } \\
\text { Gemeindebezirken }\end{array}$ & $\begin{array}{l}\text { Grün- } \\
\text { dungs- } \\
\text { jahr }\end{array}$ & $\begin{array}{l}\text { Akteurs- } \\
\text { typ }\end{array}$ & $\begin{array}{l}\text { Volu- } \\
\text { men }\end{array}$ & $\begin{array}{l}\text { öffentliche } \\
\text { Förderung }\end{array}$ & $\begin{array}{l}\text { angebotene } \\
\text { Kunstsparten }\end{array}$ \\
\hline \multicolumn{6}{|l|}{ XVI. Bezirk } \\
\hline Brunnenpassage & 2007 & $\begin{array}{l}\text { institutio- } \\
\text { nell }\end{array}$ & groß & hoch & $\begin{array}{l}\text { Musik, Tanz, } \\
\text { Literatur, Film }\end{array}$ \\
\hline Das Werk & 2010 & privat & groß & niedrig & $\begin{array}{l}\text { Musik, Video, } \\
\text { Theater, Literatur, } \\
\text { Bildende Kunst }\end{array}$ \\
\hline MASC Foundation & 1999 & privat & klein & niedrig & Bildende Kunst \\
\hline Ragnarhof & 2005 & privat & groß & niedrig & $\begin{array}{l}\text { Musik, Theater, Lite- } \\
\text { ratur, Bildende Kunst }\end{array}$ \\
\hline Soho in Ottakring & 1999 & privat & groß & hoch & $\begin{array}{l}\text { Bildende Kunst, Lite- } \\
\text { ratur, Theater, Tanz, } \\
\text { Musik, Film }\end{array}$ \\
\hline \multicolumn{6}{|l|}{ XIX. Bezirk } \\
\hline $\begin{array}{l}\text { Döblinger Kultur- } \\
\text { gemeinde }\end{array}$ & 1979 & privat & klein & niedrig & Musik, Tanz \\
\hline KulturQuartier & 2006 & privat & klein & niedrig & Literatur, Musik \\
\hline Local & 2008 & privat & groß & niedrig & Musik \\
\hline $\begin{array}{l}\text { Neues Theater in } \\
\text { Döbling }\end{array}$ & 2005 & privat & klein & niedrig & Theater \\
\hline Theater zum Himmel & 2008 & privat & groß & niedrig & Theater \\
\hline \multicolumn{6}{|l|}{ XXI. Bezirk } \\
\hline Blue Simon/ Davis & 2006 & privat & klein & niedrig & Musik \\
\hline Forum 21 & 1985 & $\begin{array}{c}\text { institutio- } \\
\text { nell }\end{array}$ & klein & niedrig & $\begin{array}{l}\text { Literatur, Bildende } \\
\text { Kunst }\end{array}$ \\
\hline Gloria Theater & 2001 & privat & groß & hoch & Theater \\
\hline $\begin{array}{l}\text { Hollywood Megaplex } \\
\text { im SCN }\end{array}$ & 1999 & $\begin{array}{l}\text { institutio- } \\
\text { nell }\end{array}$ & groß & niedrig & Film \\
\hline $\begin{array}{l}\text { Kulturverein Trans- } \\
\text { danubien }\end{array}$ & 1998 & privat & klein & niedrig & $\begin{array}{l}\text { Literatur, Kabarett, } \\
\text { Musik }\end{array}$ \\
\hline
\end{tabular}

Quelle: Eigene Zusammenstellung

Tab. 1: Merkmale der im XVI., XIX. und XXI. Wiener Gemeindebezirk untersuchten Kultureinrichtungen 
Bezirk, in Wien, in Österreich und mit Partnern im Ausland. Am häufigsten sind hier simultane Kooperationen auf verschiedenen räumlichen Ebenen. Mit Partnern im Ausland arbeiten beispielsweise „Soho“ und die „MASC Foundation“ zusammen.

Parallel zur Gründung neuer stationärer Kultureinrichtungen in den Außenbezirken wurden in den vergangenen Jahren in Wien mehrere Kulturfestivals ins Leben gerufen, die Spielorte in den Außenbezirken einbeziehen und damit einen wesentlichen Beitrag zur Kultur am Stadtrand liefern. Elf der zwölf untersuchten Veranstaltungsreihen entstanden in den 2000er Jahren. Seiner Vorreiterrolle verdankt das bereits 1990 gegründete „Volxkino“ die Eingliederung in den Kanon der Kulturfestivals. Die Hälfte aller Festivals ist an internationalen Musikstilen ausgerichtet. Insgesamt widmen sich zwei Drittel der Veranstaltungsreihen der Musik. Jeweils ein Festival ist mit der Literatur, der Bildenden Kunst und dem Film befasst. „Salam Orient“ ist das einzige Festival, das mehrere Kunstrichtungen präsentiert.

Mit der vom Echomedia-Verlag gegründeten „Kriminacht“ und dem vom Wiener Volksliedwerk ins Leben gerufenen Festival „Wean Hean“ wurden zwei der zwölf Veranstaltungsreihen von institutionellen Akteuren etabliert. Für die Gründung von zehn Festivals zeichnen private Akteure verantwortlich.

Die Festivals bieten in unterschiedlichem Ausmaß Veranstaltungen in den Wiener Außenbezirken an. Reihen mit dem höchsten Anteil von Präsentationen in den Randbezirken sind das „Internationale Akkordeonfestival“, das „Klezmore-Festival“, der „Musikalische Adventkalender“, der „Vienna Blues Spring“, das „Vienna Club Festival“ der „IG Jazz“, „Voicemania“, das „Volxkino“ und „Wean Hean“. Einige Festivals wie „Balkan Fever“ oder die „Kriminacht“ verringerten in den vergangenen Jahren ihre Präsenz in den Außenbezirken.

\section{4 Übergeordnete Fragen zur Kultur am Wiener Stadtrand}

Am Beispiel dieser Projekte treten unserem Befund nach die folgenden generellen Tendenzen zutage: Ein erster in den Wiener Außenbezirken zu beobachtender Trend ist die Revitalisierung alter Veranstaltungssäle. Rezente Beispiele dafür sind das „Casino Baumgarten“ (XIV. Bezirk), das „Gschwandner“ (XVII.) und die „Residenz Zögernitz“ (XIX.), die nun wieder für kulturelle Zwecke genutzt werden.

Eine zweite Tendenz zeigt sich in den in jüngster Vergangenheit von Immobilienentwicklern lancierten Kulturprojekten. Die Ankerbrotfabrik ist das renommierteste dieser Unternehmen. Die Loft City GmbH \& Co. KG hat einen großen Teil des Areals der Ankerbrotfabrik im X. Bezirk erworben und umgebaut. Die Inwertsetzung zielt sowohl auf Lofts ${ }^{2}$ für Unternehmer aus den Creative Industries als auch auf eine kulturelle Nutzung im engeren Sinn ab. In der früheren Expedithalle der Brotfabrik finden größere Events statt, und mit „Hilger Contemporary“, der Galerie „Ostlicht“

2) Zur Wohnung umfunktionierte Lager- oder Industrieräume 
und dem „Atelier 10“ der Caritas haben sich bereits mehrere Kulturprojekte in der Ankerbrotfabrik angesiedelt. Somit bildet die Ankerbrotfabrik einen neuen Hotspot im bislang kulturell unterversorgten X. Bezirk (Imlinger 2012; Loft City 2014; SPIEGLER 2011). Ein weiteres derartiges Projekt ist das von JP Immobilien erworbene „Gschwandner“ im XVII. Bezirk, das nun ebenfalls für Kulturveranstaltungen und ähnliche Events genutzt wird (Gschwandner 2014; Sснuн 2012). Der beschriebene Trend kommt aus den USA. Bis dato haben Künstler mit ihren Projekten einen nicht honorierten Beitrag zur Aufwertung von Liegenschaften und Stadtteilen geleistet. Nun nehmen das die Immobilienunternehmen selbst in die Hand.

Aus räumlicher Sicht kommt der Frage, in welchen Settings in den Wiener Außenbezirken bevorzugt neue Kulturprojekte entstehen, große Bedeutung zu. Wie die bisherigen Ausführungen belegen, sind dies vor allem die Gebiete entlang des Westgürtels (XV. bis XIX. Bezirk). Das beruht vor allem darauf, dass um die Jahrtausendwende in diesen dicht bebauten Gebieten mit gründerzeitlichem Baubestand günstige Bedingungen für die Ansiedlung von Kulturprojekten bestanden. Dieses Forschungsergebnis kann dahingehend generalisiert werden, dass Gebiete mit kompaktem Altbaubestand bessere Voraussetzungen für die Gründung von Kulturinitiativen bieten als locker verbaute Neubaugebiete.

Von Relevanz sind darüber hinaus die Fragen nach der Funktion und den Einzugsgebieten der Kultureinrichtungen. Wie das Beispiel der drei Wiener Untersuchungsbezirke zeigt, weisen die Kulturinitiativen diesbezüglich Unterschiede auf. Manche Projekte bedienen vor allem das lokale Publikum, andere ziehen Besucher aus ganz Wien an. Dazu kommen Mischformen. Pointiert formuliert lautet die Fragestellung Grätzelkultur $^{3)}$ versus überörtliche Funktion.

Zunächst zur ersten Funktion von Kulturprojekten, der Grätzelkultur. Die Stadt Wien verwendet in diesem Zusammenhang die Begriffe kulturelle Nahversorgung, Kultur vor der Haustür und Bezirkskultur (GESChäFTSGRUPPE Kultur UND W ISSENSCHAFT 2005, S. 11). In Frankreich wird dafür die Bezeichnung ,culture de proximité“ (Kultur der Nähe) gebraucht. Sicher soll der Begriff Grätzelkultur die intendierte Nähe repräsentieren. Mit den Orten der Grätzelkultur sind jedoch Kultureinrichtungen gemeint, die durchaus ein gewisses künstlerisches Niveau aufweisen. Keinesfalls ist darunter eine „bodenständige“ Kultur zu verstehen.

Angesichts der rasch voranschreitenden Globalisierungstendenzen und der Wirtschaftskrise benötigt wohl jeder Mensch eine gewisse Verankerung, Nähe, Geborgenheit und Sicherheit sowie eine Identifikation mit dem näheren Wohnumfeld (CAMARTIN 2007). Diese Nähe repräsentieren zunächst bekannte und vertraute Menschen, die im Umfeld leben. Dann entspricht dem auch eine fußläufig erreichbare Infrastruktur von wesentlichen Dienstleistungs- und Versorgungseinrichtungen. Zur Vermittlung von Verankerung, Nähe und Geborgenheit können lokale Kultureinrichtungen einen wesentlichen Beitrag leisten. Diese Aufgabe nehmen vor allem kleinere Projekte wie

\footnotetext{
3) Als Grätzel werden in Wien gefühlte sozialräumliche Einheiten in der Größe von zumeist nur mehreren Baublöcken bezeichnet.
} 
die „Döblinger Kulturgemeinde“, das „Neue Theater in Döbling“ (XIX.), das „Forum 21 “ und der „Kulturverein Transdanubien“ (XXI.) wahr.

Den zweiten Typ von Kulturprojekten vertreten Einrichtungen, die eine über das unmittelbare Umfeld oder den Bezirk hinausgehende Funktion erfüllen. Das sind zumeist größere Projekte wie der „Ragnarhof“ (XVI.) oder das „Local“ (XIX.), die ein Publikum aus allen Wiener Bezirken ansprechen und zumeist durch ein vergleichsweise avanciertes künstlerisches Programm gekennzeichnet sind. Durch die aus verschiedenen Stadtteilen erfolgende Anreise der Veranstaltungsbesucher entsteht ein gewisser Wiener Kulturbinnentourismus.

Ein etwas anders gelagerter Trend zur Diversifizierung des Publikums ist bei den Kulturinitiativen „Soho in Ottakring“ (XVI.) und „Aktionsradius Wien“ (XX.) zu beobachten. Beide Einrichtungen führen seit einiger Zeit im gesamten Stadtraum Veranstaltungen durch. Dadurch kommt es zu einem partiellen Exodus aus dem jeweiligen Stadtteil, in dem das Projekt entstanden ist.

Ein weiterer wesentlicher Aspekt ist die Orientierung der Kulturinitiativen an gesellschaftspolitischen Themen. Am stärksten setzen sich in ihren Programmen das „Palais Kabelwerk“ (seit Herbst 2014 „Werk X“) im X. Bezirk, „Soho in Ottakring“ und der „Aktionsradius Wien“ mit Soziokultur") auseinander. In Ansätzen gilt das auch für den „Ragnarhof“. Aufgrund der Befassung mit Fragen der kulturellen Diversität und der Integration ist auch die „Brunnenpassage“ (XVI.) diesem Typus zuzuordnen. Darüber hinaus widmen sich die Festivals „Balkanfever“, „Musikalischer Adventkalender“ und „Salam Orient“ dem Kulturaustausch.

Als letzter Punkt soll der bei den Kultureinrichtungen in den Wiener Außenbezirken zu konstatierende Wandel angesprochen werden. Er ist zwar nicht besonders stark ausgeprägt, vollzieht sich aber dennoch in einem gewissen Ausmaß. In den vergangenen Jahren mussten unter anderem das „Architekturzentrum West“ (XV.), die Galerie „Art \& Weise“ (XVI.), das „Theater des Augenblicks“ (XVIII.), die Galerie „purpur 19“ (XIX.) und das „Heizhaus Stammersdorf“ (XXI.) ihren Betrieb einstellen. Dies weist auf die schwierigen Bedingungen der künstlerischen Produktion in den Wiener Randbezirken hin.

\section{Kultur im 20. Pariser Arrondissement}

Der im Osten der französischen Metropole situierte 20. Stadtbezirk Ménilmontant wurde für eine Pariser Fallstudie ausgewählt. Mit knapp 200.000 Einwohnern gehört das im Jahr 1860 eingemeindete 20. Arrondissement zu den einwohnerstärksten Pariser Bezirken. Der für den Bezirk ausgewiesene Ausländeranteil liegt über und das Ausbildungsniveau der Bezirksbewohner unter dem Pariser Durchschnitt. Seit Beginn des

4) Soziokultur verbindet kulturelle mit sozialen und politischen Aspekten. 


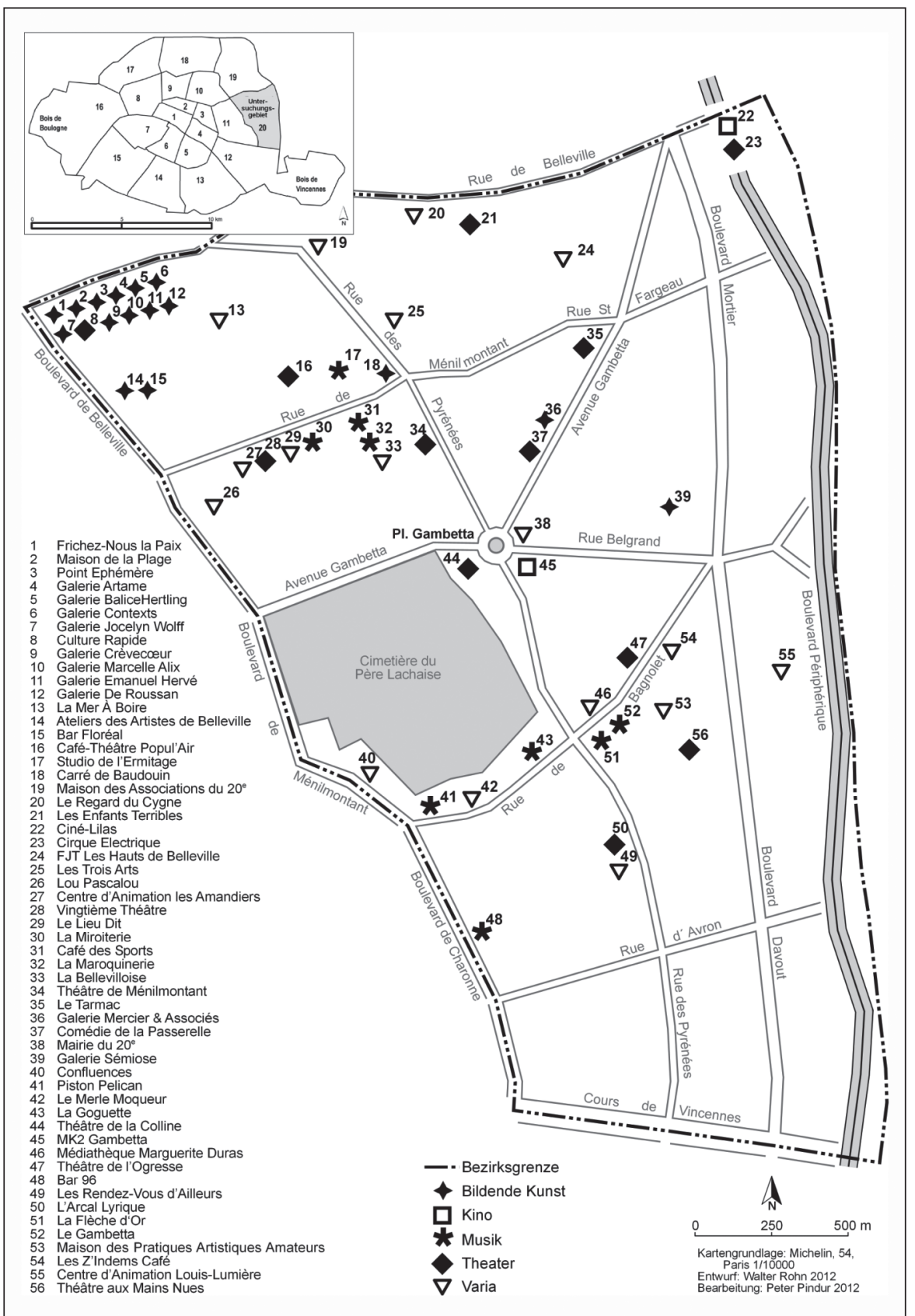

Quelle: Eigene Zusammenstellung auf Basis von Veranstaltungsankündigungen in Pariscope, Lylo, Gazette du $20^{\mathrm{e}}$ usw. und im Internet sowie von Recherchen vor Ort

Abb. 2: Kultureinrichtungen im 20. Pariser Stadtbezirk mit regelmäßigem Programm (2012) 
21. Jhs. ist der Bezirk, der im Vergleich zu den zentralen Pariser Bezirken eine schwächer ausgeprägte kulturelle Infrastruktur aufweist, durch eine sich rasch entwickelnde Kulturszene geprägt. Der 20. Pariser Stadtbezirk beherbergt insgesamt 56 traditionelle und rezente Kultureinrichtungen - zehn Theater, eine Theatergruppe und einen Zirkus, zwei Mehrsaalkinos, neun Musiklokale, 16 Galerien und Ausstellungsräume sowie 17 Einrichtungen mit gemischtem Veranstaltungsprogramm (vgl. Abb. 2).

Mit dem Galerienviertel im Nordwesten des Arrondissements, den in der Nähe der Rue de Ménilmontant und in der Rue Boyer angesiedelten Kulturinitiativen sowie den Locations in der Rue de Bagnolet weist der Bezirk drei Cluster von Kultureinrichtungen auf. Künstlerische und kulturelle Leuchttürme des Bezirks sind das Kulturzentrum „Carré de Baudouin“, das Nationaltheater „La Colline“, das dem frankophonen Theater gewidmete „Tarmac" und das „Vingtième Théâtre“, die beiden Kinos „MK2 Gambetta“ und „Ciné Lilas“, die „Médiathèque Marguerite Duras“, die multidisziplinäre „Bellevilloise“, die Musiklokale „Flèche d'Or“ und „Maroquinerie“ sowie das „Haus der Amateurkünste“. Im 20. Arrondissement sind viele private Akteure wie Künstler, Gewerbetreibende, Bezirksbewohner usw. tätig. Dazu kommt eine starke Präsenz institutioneller Handlungsträger.

Die Stadt Paris hat in den vergangenen Jahren im 20. Bezirk großes kulturelles Engagement gezeigt und mit dem „Carré de Baudouin“, der „Médiathèque Marguerite Duras“ und dem „Haus der Amateurkünste“ drei bedeutende Einrichtungen eröffnet. Die Bezirksverwaltung wendet pro Einwohner einen Euro für lokale kulturelle Aktivitäten auf und betreibt eine ambitionierte Politik zur Förderung der lokalen Kulturszene.

\section{Wiener und Pariser Randbezirke im Vergleich}

Beim Vergleich der Wiener und Pariser Untersuchungsbezirke und der Kulturpolitik der Metropolen Wien und Paris zeigen sich die folgenden Ähnlichkeiten und Unterschiede:

Das Gros aller in den drei Wiener Bezirken Ottakring, Döbling und Floridsdorf bestehenden Kulturprojekte sind kleine Initiativen mit geringer öffentlicher Förderung, die primär der Grätzelkultur entsprechen. Größere Einrichtungen, die ein Publikum aus ganz Wien ansprechen, gibt es nur wenige. Auch einer guten öffentlichen Förderung können sich nur wenige Projekte erfreuen. Der Großteil der Einrichtungen wurde von privaten Akteuren wie Künstlern, Protagonisten von Kulturinitiativen und Bezirksbewohnern gegründet. Die Mehrheit der Kulturprojekte ist dem Typ einer Off-Szene zuzuordnen - viele Initiativen strahlen den Charme des Amateurhaften und Improvisierten aus. Durch die Fluktuation der Projekte ist in der Kulturszene der drei Bezirke auch ein gewisser Wandel zu verzeichnen. Der XVI. Wiener Gemeindebezirk zeigt mit dem von Bezirksvorsteher Prokop geprägten Slogan „Kulturbezirk Ottakring" ein großes einschlägiges Engagement und weist eine beachtliche lokale Kulturförderung auf. Die beiden anderen Wiener Untersuchungsbezirke räumen der 
Kultur einen vergleichsweise geringeren Stellenwert ein. In ihrer Kulturpolitik setzt die Stadt Wien einige bemerkenswerte Initiativen für die Stadtrandbezirke, der Fokus der kulturellen Aktivitäten liegt jedoch deutlich bei den Innenbezirken.

Im Vergleich zu den drei Wiener Untersuchungsbezirken verfügt das 20. Pariser Arrondissement Ménilmontant über eine stärker entwickelte Kulturszene mit einer ausgeprägten Präsenz öffentlicher Akteure. Neben kleinen Projekten beherbergt der Bezirk auch viele große Kultureinrichtungen. Als Beispiele für Letztere seien hier zunächst einige von der öffentlichen Hand finanzierte Einrichtungen genannt: Das als Bezirkstheater fungierende "Vingtième Théâtre" wird von der Stadt Paris alimentiert. Das frankophone Theater „Tarmac“ erhält Fördermittel vom Kulturministerium, der Region, der regionalen Kulturverwaltung und der Stadt. Das Nationaltheater „La Colline“ wird ebenfalls vom genannten Ministerium finanziert. In den vergangenen Jahren hat die Stadt Paris im 20. Bezirk drei hochrangige neue Kultureinrichtungen geschaffen. Darüber hinaus weist der Bezirk eine avancierte private Theater-, Musikund Galerienszene auf. Die weitgehend auf Musik spezialisierten Kulturinitiativen „Bellevilloise“, „Flèche d'Or“ und „Maroquinerie“ gelten als Pariser Top-Einrichtungen. Die von den Kultureinrichtungen des 20. Bezirks gezeigten Produktionen sind in der Regel durch ein höheres künstlerisches Niveau geprägt als die ihrer Wiener Pendants. Insgesamt ist auch eine größere Professionalität der Häuser zu verzeichnen.

Im Vergleich zu den Wiener Untersuchungseinheiten ist die Kulturszene des 20. Pariser Bezirks einem rascheren Wandel unterworfen. Beim Stellenwert des 20. Arrondissements ist jedoch auch die zweifache Positionierung des Bezirks am Rand der Hauptstadt Paris und im Zentrum einer großen Agglomeration zu berücksichtigen.

Gegenüber Wien ist die Kulturpolitik der Stadt Paris stärker territorial ausgerichtet und setzt Schwerpunkte in benachteiligten Stadtvierteln. Die Pariser Stadtverwaltung strebt eine ausgewogenere Verteilung der kulturellen Ressourcen und des kulturellen Angebots im Stadtgebiet an. Insgesamt zeigt die Stadt Paris ein wesentlich stärkeres Engagement in den Peripheriebezirken als Wien in seinen Außenbezirken.

\section{Effekte von Kulturprojekten auf die Entwicklung peripherer Stadtteile}

Nach Bianchini \& GHiLARdi (2004) können kulturelle Initiativen - über ihre Rolle als Kunstproduzenten und Veranstalter hinaus - die Funktion von Katalysatoren der Stadtentwicklung übernehmen. Im Anschluss an die Untersuchung von Kultureinrichtungen an den Rändern europäischer Metropolen wurden die möglichen Auswirkungen der Initiativen auf die kulturelle, städtebauliche, wirtschaftliche und soziale Entwicklung peripherer Stadtviertel analysiert. Für die Auseinandersetzung mit diesem sowie mit zwei weiteren Themenbereichen wählte der Autor die Methode der Expertenbefragung. In diesem Kontext wurden in Wien fragebogenbasierte Interviews mit drei Trägern politischer Funktionen, vier Experten für Stadtentwicklung, drei Kulturfachleuten sowie drei Proponenten von Kulturinitiativen geführt. 
Am Beginn der Gespräche standen die Aufgaben und Perspektiven der Kulturinitiativen im Mittelpunkt. Für die nächsten Jahre rechnen die Experten mit der Gründung weiterer Kulturinitiativen am Stadtrand. Eine Zusammenballung derartiger Einrichtungen wird nur für kleinflächige Bezirke prognostiziert. Positive Auswirkungen der Kulturinitiativen erwarten die befragten Fachleute zunächst für den Ausbau der kulturellen Infrastruktur der Randbezirke. Die Mehrheit der Gesprächspartner nimmt an, dass die Initiativen sowohl überörtliche als auch lokale Funktionen erfüllen können. Eine spezielle Aufgabe wird den Kulturprojekten im Sinne der Nachbarschaftskultur zugewiesen. Als wesentlich wird auch der Beitrag der Kulturinitiativen zur Demokratisierung von Kultur angesehen. Diese Zielsetzung wird von den Gesprächspartnern in den breiteren Rahmen der Kommunikation, der Bildung von Beziehungsgeflechten unter den Bewohnern eines Stadtviertels und der Integration gestellt.

Die Beiträge der Kulturinitiativen zur städtebaulichen Entwicklung der Randbezirke, zur Aufwertung von Stadtvierteln, zur Verbesserung der Lebensbedingungen und zur Erhöhung der Wohnzufriedenheit sind weitere von den Experten sehr positiv bewertete Funktionen der Projekte. In Anlehnung an das Konzept der kulturellen Planung wird von den Interviewpartnern eine Verknüpfung kultureller Aktivitäten mit anderen politischen Handlungsfeldern vorgeschlagen, die zu positiven Wechselwirkungen führen soll. Besonders hervorgehoben wird die Rolle der in den Randbezirken tätigen Personen und Gruppen, der Akteure. Als Beispiele für Träger von kulturellen Aufwertungsprozessen in den Außenbezirken werden häufig „Soho in Ottakring“ und die weiteren im Bezirk angesiedelten Kulturinitiativen sowie der „Aktionsradius Wien“ im Augartenviertel des XX. Bezirks genannt. Einige Experten weisen darauf hin, dass zur Erfüllung der angeführten Aufgaben eine gewisse Größe der Kultureinrichtungen oder eine bestimmte Menge derartiger Projekte erforderlich ist. In den Gesprächen klammern die Experten nicht aus, dass kulturelle Aufwertungsprozesse letzten Endes den Verwertungsinteressen von Grundeigentümern und Bauträgern dienen. Wie in vielen nordamerikanischen und westeuropäischen Städten zu beobachten ist, treten die Künstler häufig als unbedankte Pioniere der Gentrifizierung auf.

Eine weitere wichtige Funktion der Kulturinitiativen besteht nach Einschätzung der Interviewpartner in der Stimulierung der lokalen Ökonomie. Im Einzelnen können Kulturprojekte mittelbar oder unmittelbar zur Generierung von Arbeitsplätzen beitragen, die Wertschöpfung der lokalen Kreativwirtschaft fördern und die Reanimation von Geschäftsvierteln in den Außenbezirken unterstützen.

Besonders hoch eingeschätzt wird von den befragten Experten die Rolle der Kulturprojekte bei der Förderung von Integration und Partizipation. In diesem Zusammenhang werden die Beiträge der Kultureinrichtungen zur Herstellung eines Klimas der Offenheit, zur Intensivierung der Partizipation und zum Empowerment der lokalen Bevölkerung als bedeutend angesehen. Im Sinne der Partizipation wird die Beteiligung lokaler (kultureller) Initiativen an Planungs- und Entscheidungsprozessen vorgeschlagen. Ein gutes Beispiel für die von den Kulturprojekten zu erfüllenden Aufgaben sind die Freiluftvorführungen des „Volxkinos“, die eine Auseinandersetzung mit dem öffentlichen Raum ermöglichen.

Sehr positiv gesehen werden von den Gesprächspartnern auch die Beiträge der Kulturinitiativen zur Stärkung der Identität von Stadtteilen, zur Intensivierung der 
Identifikation der Bewohner mit ihrem Stadtviertel und zum Generieren neuer Images für periphere Stadtteile. Am Beispiel des „Palais Kabelwerk“ im XII. Bezirk wird die besondere Bedeutung der Identitätsstiftung für Neubauviertel hervorgehoben. Die Experten bezeichnen die Kulturinitiativen als Landmarks, die einem Stadtteil erst Identität verleihen. Neben der Identitätsstiftung könnten Kulturprojekte auch dazu beitragen, eine entsprechende Atmosphäre für einen Ort zu kreieren. Es wird auch darauf hingewiesen, dass die Herausbildung einer prononcierten Identität möglicherweise einen längeren Zeitraum in Anspruch nehmen kann. Die Gesprächspartner betonen, dass die Identifikation der Menschen mit ihrem Wohnumfeld und die Herausbildung von neuen Vorstellungsbildern für Stadtteile nur durch originäre Strategien gefördert werden können. Große Bedeutung kommt hier der Nachhaltigkeit der einzelnen Projekte zu.

Insgesamt belegt die Expertenbefragung, dass Kulturinitiativen eine Reihe wertvoller Impulse für die Stadtentwicklung setzen können.

\section{Kulturpolitik für städtische Randzonen}

Nach den Effekten der Kulturinitiativen wurden in einem nächsten Schritt die gegenwärtige Unterstützung der Stadt Wien für Peripheriebezirke und dezentrale Kulturinitiativen analysiert sowie die Umrisse einer möglichen Kulturpolitik für städtische Randzonen gezeichnet.

Die befragten Experten identifizieren spezifische Förderschienen wie „Kunst im öffentlichen Raum“, das Bezirkskulturfestival „Wir sind Wien“, das Festwochenprogramm „Into the City“, die Jugendaktion „Cash for Culture“, die Direktförderung einzelner Projekte durch die Kulturabteilung der Stadt Wien und die Kulturbudgets der Bezirke. Die Mehrheit der Gesprächspartner gibt jedoch zu Protokoll, kein ausgewiesenes Programm der Stadt Wien für die Kultur am Stadtrand zu kennen. Vor diesem Hintergrund befürwortet das Gros der Fachleute die Konzeption und Umsetzung einer speziellen Politik zur Förderung von Kulturprojekten an der städtischen Peripherie.

Unterschiedliche Auffassungen vertreten die Experten bezüglich einer zentralen oder dezentralen Ausrichtung dieses Programms. Einigkeit besteht hingegen bezüglich der Notwendigkeit einer adäquaten Förderung der Kulturprojekte in den Wiener Außenbezirken.

Abschließend wurde die Frage des Lukrierens der erforderlichen finanziellen Mittel aufgeworfen. Nachdem eine deutliche Aufstockung des Kulturbudgets außerhalb des Realisierbaren zu liegen scheint, plädiert die Mehrheit der Experten dafür, die erforderlichen Ressourcen durch eine Umschichtung innerhalb des Budgets der Stadt Wien bereitzustellen. Dafür sollen nicht nur Mittel aus der Hochkultur abgezogen, sondern auch von der Stadtentwicklung und anderen Ressorts entsprechende Fonds bereitgestellt werden.

Als Ergebnis der Expertenbefragung kann ein explizites Plädoyer für eine spezielle Politik zur Förderung der Kultur am Stadtrand festgehalten werden. 


\section{Förderung dezentraler Kulturinitiativen}

Die Umsetzung der bezeichneten kulturpolitischen Strategie bedarf erstens einer politischen Vision - nämlich jener, die Kultur an der Peripherie stärker zu fördern und zweitens geeigneter Maßnahmen. Zu letzteren entwarf der Autor acht spezielle Zielsetzungen und legte diese den Interviewpartnern zur Beurteilung vor.

Die erste zur Diskussion gestellte Maßnahme bezog sich auf die Implantierung von kulturellen Leitprojekten wie Museen, Konzerthallen usw. in den Wiener Außenbezirken. Für diesen Vorschlag fand sich eine knappe Mehrheit der befragten Experten.

Die zweite Frage lautete, ob in den Randbezirken bestimmte Kunstsparten besonders forciert werden sollen. Dabei sah sich die Mehrheit der Gesprächspartner nicht in der Lage, besonders für die Außenbezirke geeignete Kunstgattungen zu identifizieren.

Der dritte unterbreitete Vorschlag zielte auf die explizite Förderung der Jugendkultur - z.B. Hip-Hop, Slam, Break Dance, Graffiti usw. - in den Randbezirken ab. Diese Maßnahme wurde von einer deutlichen Mehrheit der Experten für gut befunden.

Von einer großen Mehrheit der Befragten befürwortet wurde auch die vierte vorgeschlagene Fördermaßnahme, die Bereitstellung von geeigneten Räumen für dezentrale Kulturinitiativen durch die Stadt Wien. In diesem Kontext wurde auch ein Relaunch der Häuser der Begegnung diskutiert.

Der fünfte, ebenfalls von einer deutlichen Majorität begrüßte Vorschlag bestand in der Einrichtung eines Expertenpools für dezentrale Kulturprojekte.

Ein leichtes Übergewicht der Befürworter gab es bei der sechsten Maßnahme, der Unterstützung der Netzwerkbildung und des Clusterings von Kulturinitiativen durch die Stadtverwaltung.

Auf sehr große Zustimmung stieß die als Punkt sieben vorgeschlagene Förderung von Public-Relations-Aktivitäten der Initiativen durch die Stadt Wien.

Die achte Frage bezog sich auf die Möglichkeit des Gewinnens weiterer öffentlicher Geldgeber sowie privater Sponsoren für Kulturprojekte in den Wiener Außenbezirken. Dies hält eine knappe Mehrheit der Experten für möglich. Der neueste Trend geht hier in Richtung Crowdfunding.

Der Großteil der vorgeschlagenen Maßnahmen könnte also zur Definition der Konturen einer Kulturpolitik und einer kulturpolitischen Strategie für städtische Randzonen dienen.

\section{Schluss}

Wie der langjährige Pariser Kulturstadtrat Christophe GIRARD im Gespräch ausführte, organisiert sich rund um eine kulturelle Aktivität, die man setzt, ein ganzes Leben. Nach Auffassung des Autors ist dies der Blickwinkel, aus dem Kulturinitiativen am Stadtrand primär betrachtet werden sollten. Über die von den Experten diskutierten Effekte hinaus können Kultureinrichtungen weitere wesentliche Akzente setzen.

Die zentralen Kultureinrichtungen in Wien stehen theoretisch allen Bewohnern der Stadt offen. Dennoch stellt die Entfernung zwischen dem Wohnort und den gro- 
ßen Bühnen, Konzertsälen und Museen im Stadtzentrum einen wesentlichen Faktor der Zugänglichkeit dar. Durch die Bereitstellung einer entsprechenden kulturellen Infrastruktur in den Randbezirken werden die Anfahrtswege der Besucher zu den Kultureinrichtungen verkürzt oder sie entfallen ganz. Im Sinne einer Stadt der kurzen Wege kann damit auch ein wertvoller Beitrag zum Umweltschutz geleistet werden.

Gemäß dem Konzept der Kultur der Nähe können lokale Kulturinitiativen den Menschen eine Verankerung im unmittelbaren Wohnumfeld bieten sowie Geborgenheit und Sicherheit vermitteln. Das ist vor allem in Neubaugebieten wesentlich. Nach Auffassung des Autors kommt dabei soziokulturellen Projekten ein hoher Stellenwert zu. Beispiele für Initiativen, die eine Verbindung von Kultur und Politik herstellen, sind für Wien das „Palais Kabelwerk“, ,Soho in Ottakring“ und der „Aktionsradius Wien“ und für Paris „Lieu-dit“ und „Confluences“. In diesem Zusammenhang können Kulturinitiativen auch als Anlaufstellen für die Artikulation gesellschaftlicher Anliegen dienen. Darunter sind sowohl Fragen, die die Lebensbedingungen im jeweiligen Stadtviertel betreffen, als auch allgemeine politische Themen zu verstehen. Besonders vor dem Hintergrund der Wirtschaftskrise kommt dezentralen Kulturinitiativen, die eine enge Kooperation mit Partnern im lokalen Umfeld pflegen, eine nicht zu unterschätzende Bedeutung zu.

Im angesprochenen Kontext ist es wesentlich, jene Elemente der Kunst zu fördern, die über die wirtschaftlichen Verwertungszusammenhänge hinausreichen und Kunstströmungen zu forcieren, die sich der konkreten Lebensverhältnisse der Menschen annehmen (vgl. Metz \& SEesslen 2014). Ein hervorragendes Beispiel für dezentrale politische Kulturarbeit bildet das Wiener Gemeindehoftheater der 1980er und 1990er Jahre. Unter der Leitung von Didi Macher bespielte das auch als „Dario-Fo-Theater in den Arbeiterbezirken“ bezeichnete Ensemble vor allem die Höfe von kommunalen Wohnbauten (PFEIFFER 2009). Ein gutes Beispiel für kontemporäres politisches Theater ist die Arbeit der Theatergruppe „13. Januar“. Im Juli 2011 präsentierte das Ensemble in Grammatneusiedl (Niederösterreich) eine mit aktuellen Bezügen versehene Bühnenfassung der klassischen soziologischen Studie „Die Arbeitslosen von Marienthal“. Die im Rahmen des Viertelfestivals Niederösterreich am Originalschauplatz gezeigte Aufführung trug den Titel „Guter Morgen Marienthal“ (Kulturwoche 2012). Als dritten Modellfall soll auf eine rezente Veranstaltungsreihe in Graz hingewiesen werden. Unter dem Motto ,Truth Is Concrete - die Wahrheit ist konkret“ - setzte sich das Festival „Steirischer Herbst“ im Jahr 2012 mit „,künstlerischen Strategien in der Politik“ und ,politischen Strategien in der Kunst“" auseinander (STEIRISCHER Herbst 2012; Steirischer Herbst \& Malzacher 2014).

Zum Schluss ein kurzes Plädoyer: In den Wiener Außenbezirken, den Bezirken $\mathrm{X}$ bis XXIII, leben drei Viertel der Wiener Bevölkerung (Magistrat der Stadt Wien 2014). In diesem Raum wird jedoch nur ein kleiner Teil des Kulturbudgets der Stadt Wien veranlagt. Im Sinne des von Henri Lefebvre (2009) formulierten Rechts auf Stadt hat die große Mehrheit der Wiener Anspruch auf entsprechende kulturelle Infrastruktur. Deshalb sei empfohlen, die vorhin formulierte kulturpolitische Strategie für die Wiener Außenbezirke umzusetzen und so für eine egalitärere Verteilung der kulturellen Ressourcen zu sorgen. 


\section{Literaturverzeichnis}

Bianchini F., Ghilardi L. (2004), The Culture of Neighbourhoods: A European Perspective. In: Bell D., JAYNe M. (Hrsg.), City of Quarters: Urban Villages in the Contemporary City, S. 237-248. Aldershot, Ashgate.

Camartin I. (2007), Heimat. Schriftenreihe Vontobel-Stiftung 1770. Zürich, Vontobel-Stiftung. Evans G. (2001), Cultural Planning: International Perspectives. London - New York, Routledge. Fuchs M. (2007), Kulturpolitik. Wiesbaden, Verlag für Sozialwissenschaften.

Geschäftsgruppe Kultur und Wissenschaft des Magistrats der Stadt Wien (Hrsg.) (2005), Kunst- und Kulturbericht der Stadt Wien 2004 - Frauenkulturbericht. - http://www. wien.gv.at/kultur/abteilung/pdf/kunstbericht2004a.pdf (falls nicht anders angegeben alle Zugriffe per 25.6.2014).

Greffe X., Pflieger S. (2005), La culture et le développement local. Paris, Éditions de l'OCDE. Gschwandner (Hrsg.) (2014), *gschwandner. - http://www.gschwandner.at/

ImLinger C. (2012), Atelier 10: Kunst statt „Mitleids-Getue“. In: Die Presse, 29.7.2012. - http://diepresse.com/home/leben/mensch/1271512/Atelier-10_Kunst-statt-Mit leidsGetue?from=suche.intern.portal

KLeIN A. (2005), Kulturpolitik. Eine Einführung. Wiesbaden, Verlag für Sozialwissenschaften. Konrad H. (2011), Kulturpolitik. Eine interdisziplinäre Einführung. Wien, Facultas/WUV.

Kulturwoche (Hrsg.) (2012), Ein Stück Arbeit: Guter Morgen Marienthal - die Premierenkritik. - http://www.kulturwoche.at/index.php?option=com_content\&task=view\&id=2780 \&Itemid $=1$

LANDRY C. (2006), The Art of City Making. London, Earthscan.

Latour B. (2007), Eine neue Soziologie für eine neue Gesellschaft. Einführung in die AkteurNetzwerk-Theorie. Frankfurt am Main, Suhrkamp.

Lefebvre H. (2009), Le droit à la ville. Paris, Economica (Erstveröffentlichung: 1968).

LiNDNER R. (2007), Die Entdeckung der Stadtkultur. Soziologie aus der Erfahrung der Reportage. Frankfurt am Main - New York, Campus.

Loft City (Hrsg.) (2014), Loft City GmbH \& CO KG. - http://www.loftcity.at/

Löw M. (2008), Soziologie der Städte. Frankfurt am Main, Suhrkamp.

LÜders C. (2009), Beobachten im Feld und Ethnographie. In: Flick U., von Kardorff E., STEINKE I. (Hrsg.), Qualitative Forschung. Ein Handbuch, S. 384-401. Reinbek bei Hamburg, Rowohlt.

MA7 - Kulturabteilung (Hrsg.) (2010), Wien Kultur. Die Geschichte wird weitererzählt. Büro des Stadtrats für Kultur und Wissenschaft. Wien.

Magistrat der Stadt Wien (Hrsg.) (2014), Bevölkerung nach Bezirken 2005 bis 2013. - http:// www.wien.gv.at/statistik/bevoelkerung/tabellen/bevoelkerung-bez-zr.html

Mairie de Paris (Hrsg.) (2011), Politique culturelle de la ville de Paris. Paris.

Metz M., Seesslen G. (2014), Geld frisst Kunst. Kunst frisst Geld. Ein Pamphlet. Berlin, Suhrkamp.

MiegGelbrinK J., Wiest K. (2009), Fachsitzung Geographentag zur komparativen Forschung. Wien mit Berlin und Äpfel mit Birnen? Raumbezogene Vergleiche in wissenschaftlichen und politischen Praktiken. - http://www.geographentag-wien.at/fileadmin/user_upload/ conf_geotag09/Download/FS-Vorschl\%C3\%A4ge/FS_42_Geotag_Miggelbrink_Wiest. pdf (23.5.2012)

Mulcahy K.V. (2006), Cultural Policy: Definitions and Theoretical Approaches. In: Educult (Hrsg.), ICCPR 2006/ Proceedings/ CD-Rom. Fourth International Conference on Cultural Policy Research, University of Music and Performing Arts, Vienna, 12.-16.7.2006. 
Municipial Cultural Planning Project (MCPP) (Hrsg.) (2003), Glossary. Municipial Cultural Planning Project. - http://www.culturalplanning.calmcpp/ib_glossary.html (26.11.2003).

Pfeiffer G.C. (2009), Kommt herbei! Comœdianten sind da. Ich erzähle Euch die Geschichte vom Dario-Fo-Theater in den Arbeiterbezirken. Eintritt frei. Wien, Mandelbaum Verlag.

Quenzel G. (Hrsg.) (2009), Entwicklungsfaktor Kultur. Studien zum kulturellen und ökonomischen Potenzial der europäischen Stadt. Bielefeld, Transcript.

Roнn W. (2003), Wien. Die neue Kultur an der Peripherie der Stadt. In: dérive, 4, 2, S. 30-31.

Rohn W. (2004), Culture and Urban Development. New Cultural Infrastructures on the Outskirts of Vienna and Paris. In: Borsdorf A., Zembri P. (Hrsg.), European Cities. Insights on Outskirts. Band 3: Structures (= Cost C10 Publications \& Papers), S. 149-167. Paris, Cost.

Roнn W. (2007), Peripherie und Kultur - zu den gesellschaftlichen Auswirkungen von Kulturinitiativen für die Entwicklung der Wiener Außenbezirke. In: SWS-Rundschau, 47, 3 , S. 321-342.

Roнn W. (2013), Die neue Kultur am Rand der Stadt: Wien und Paris. Wien, Praesens.

Schuн K. (2012), Gschwandner: Der alte Charme der Vorstadt. In: Die Presse, 5.9.2012. - http:// diepresse.com/home/panorama/oesterreich/1275688/Gschwandner_Der-alte-Charme-der -Vorstadt?from=suche.intern.portal

Skot-Hansen D. (2005), Why Urban Cultural Policies? In: Robinson J. (Hrsg.), Eurocult 21. Integrated Report, S. 31-39. Helsinki, Eurocult 21. - http://www.eurocult21.org/books/ IntegratedReport3-116screen.pdf (3.9.2009)

SpIEGLer A. (2011), Ein frischer Duft weht in der Kulturfabrik. In: Die Presse, 19.6.2011, S. 43.

STEIRISCHER Herbst (Hrsg.) (2012), Programmplaner. - http://www.steirischerherbst.at/2012/ deutsch/programm/remixer.php

Steirischer Herbst, Malzacher F. (Hrsg.) (2014), Truth is Concrete. A Handbook for Artistic Strategies in Real Politics. Berlin, Sternberg Press.

Twickel C. (2010), Gentrifidingsbums oder eine Stadt für alle. Hamburg, Edition Nautilus.

W ARD S.V. (2006), „Cities are Fun!“‘: Inventing and Spreading the Baltimore Model of Cultural Urbanism. In: Monclús J., Guàrdia M. (Hrsg.), Culture, Urbanism and Planning, S. 271-285. Aldershot, Ashgate. 